
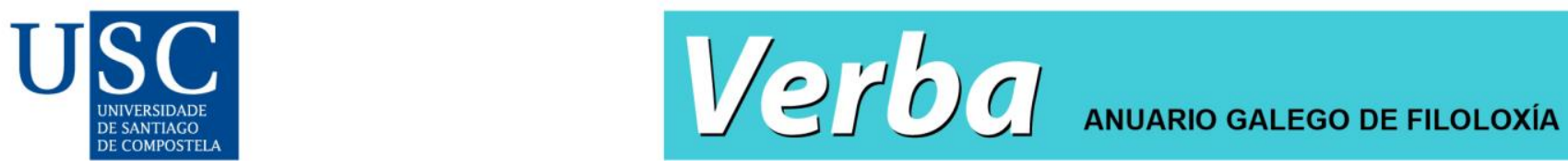

Verba: Anuario Galego de Filoloxía, 48, 2021. ISSN: 2174-4017

https://doi.org/10.15304/verba.48.6942

\title{
Comentarios críticos sobre el libro Animal languages, de Eva Meijer ${ }^{1}$ \\ Critical comments on the book Animal languages, by Eva Meijer
}

Víctor M. Longa ${ }^{1}$

1Universidade de Santiago de Compostela, España

Recibido: 17/06/2020; Aceptado: 24/08/2020

\section{Resumen}

Este trabajo efectúa un análisis crítico del libro Animal languages, escrito por la filósofa holandesa Eva Meijer. La intención de la autora es mostrar que los animales tienen destrezas cognitivas y comunicativas complejas, pero su estrategia vincula esa complejidad comunicativa con el carácter lingüístico de los códigos comunicativos animales. Más allá de sostener que los animales tienen lenguaje (algo frecuente en etólogos), la obra deja mucho que desear desde la óptica científica. El trabajo mostrará que el libro de Meijer está repleto de contradicciones, exageraciones, afirmaciones extravagantes o infundadas, tergiversaciones de la opinión de otros autores, errores en el uso de referencias bibliográficas o desconocimientos muy graves de diferentes aspectos.

Palabras clave: Eva Meijer; comunicación animal; cognición animal; lenguaje; sintaxis.

\section{Abstract}

This paper provides with a critical analysis of the book Animal languages, written by the Dutch philosopher Eva Meijer. The author aims at showing that non-human animals do have complex cognitive and communicative skills; however, her strategy links the aforementioned communicative complexity to the linguistic nature of animal communicative systems. Beyond arguing that animals are endowed with language (a quite common claim by ethologists), the book leaves much to be desired from a scientific point of view. The paper will show that Meijer's book is full of contradictions, exaggerations, bizarre or baseless claims, distortions of other authors' assertions, mistakes in the use of bibliographical references or serious ignorance of a number of aspects.

Keywords: Eva Meijer; animal communication; animal cognition; language; syntax. 


\section{INTRODUCCIÓN}

Los grandes avances en etología han mostrado incontestablemente que los animales tienen representaciones mentales y procesos cognitivos complejos (Bermúdez 2003; Budiansky 1998; Bueno-Guerra \& Amici eds. 2018; Carruthers 2006: cap. 2; Couzin 2009; Hauser 2000; Menzel \& Fisher eds. 2011; Spelke \& Lee 2012; Wynne \& Udell 2013), incluso los insectos (Chittka 2017; Gallistel 1998; Longa 2014; Reznikova 2018; Webb 2012). Esa misma complejidad rige para el ámbito comunicativo, pues múltiples estudios han revelado de modo no menos inequívoco que muchas especies tienen sistemas comunicativos muy sofisticados, con propiedades no imaginables hace medio siglo (Choe ed. 2019; Hauser 1996; Hauser \& Konishi eds. 1999; Longa 2012; Oller \& Griebel eds. 2004; para una panorámica, cfr. Longa 2013).

Muy recientemente, se ha publicado la traducción inglesa de una obra escrita en holandés por la polifacética holandesa Eva Meijer (Meijer 2019), filósofa, artista, novelista, cantante y compositora. El objetivo del libro es mostrar que los animales exhiben cognición y comunicación compleja, centrándose especialmente en el segundo plano. Como es bien sabido, desde que se empezó a advertir la complejidad de la comunicación animal, surgió una polémica, alentada por bastantes etólogos, sobre si la comunicación animal (en estado salvaje o en laboratorio) comparte propiedades con el lenguaje. Meijer (2019) se inscribe en esta tradición, sosteniendo con insistencia que la comunicación animal supone lenguaje. El libro es profundamente decepcionante, no tanto porque defienda el aspecto mencionado (en todo caso, sin mucha base), sino sobre todo porque deja mucho que desear científicamente, al estar repleto de exageraciones, afirmaciones extravagantes, tergiversación de autores manejados como fuentes, confusión en algunas referencias o graves desconocimientos impropios de una obra académica, entre otros aspectos que analizaré a continuación.

La estructura de este trabajo es la siguiente: el apartado 2 discute las tesis generales del libro de Meijer con respecto al lenguaje y a la comunicación y el 3 muestra la estrategia generalizada de la autora de tergiversar gravemente fuentes bibliográficas usadas por ella. El apartado 4 discute la inadecuada comprensión por parte de Meijer de aspectos semióticos y lingüísticos básicos, mientras que el 5 ofrece algunos ejemplos de errores conceptualmente muy graves cometidos que descalifican a Meijer, como atribuir carácter innato al reconocimiento de las llamadas maternas en patos o considerar que FOXP2 es 'el gen del lenguaje'. Finalmente, el apartado 6 ofrece una breve conclusión.

\section{LENGUAJE Y COMUNICACIÓN}

Meijer sostiene acertadamente que «the more we learn about animal communication, the more complex it appears to be» (p. 182). Sin embargo, en vez de realzar esa complejidad en sus propios términos, se embarca en un intento estéril de mostrar que los sistemas comunicativos animales presuponen lenguaje: «my starting point is that animals have language» (p. 12). De ahí que afirme, por ejemplo, que los perritos de las praderas (roedor americano) «have an extensive language» (p. 1), que pollos y cerdos también tienen «an extensive language» (p. 124), o que los elefantes cautivos «can speak in human words» (p. 1).

El libro revela la confusión entre función (conducta general) y herramienta (mecanismo concreto para satisfacer esa conducta). Todas las especies comparten las mismas funciones 
(locomoción, reproducción, alimentación o comunicación), pero cada una emplea herramientas muy diferentes (en cuanto a la locomoción, algunos seres reptan, otros vuelan, etc.). Meijer fusiona comunicación (función) y lenguaje (herramienta concreta) cuando afirma que «if you assume that animals have no language and cannot communicate meaningful [...]» (pp. 13-14), sosteniendo así que negar el lenguaje en animales supone negar que comuniquen. El uso no técnico del término 'lenguaje' se revela claramente al afirmar que «Joy the pony [...] made me realise that it is possible for humans and other animals to have an extensive shared language» (pp. ix-x) o que "there are many ways in which humans have linguistic interactions with other animals" (p. 43). Que podamos interpretar señales de un animal y viceversa no implica, obviamente, un 'lenguaje' o código común.

Por ello, no tiene sentido escribir que «from an evolutionary perspective, it would be strange for humans to have language and for other animals not to have something similar» (p. 88), como no lo tendría afirmar que, evolutivamente, sería raro que las abejas tuvieran una danza comunicativa y que los restantes seres carecieran de algo similar. La función es la misma, pero las diferencias surgen en cómo aquella se implementa. Como escribe Anderson (2004: 4-5), «human language is uniquely human, just as many complex behaviors of other species are uniquely theirs».

Sin embargo, Meijer cambia de criterio en varias ocasiones (no es el único ejemplo de tal proceder): si muchas veces proclama tajantemente el carácter lingüístico de la comunicación animal, otras veces matiza esa afirmación, indicando que las señales animales «are not exactly comparable to a natural language like Dutch or English, and yet they can certainly be interpreted as expressions of language» (p. 44) (parece aquí asumir una gradación del lenguaje, semejante a la de Rondal 2000). Pero sorprendentemente, tras insistir durante todo el libro que la comunicación animal es lenguaje, señala que «instead of deciding whether non-human animal forms of communication fit into the frame of what humans define as 'language', we should instead pay attention to what they are saying, and begin investigating what language is and could be from there» (p. 216). ¿En qué quedamos?

El libro esta repleto de afirmaciones extravagantes ${ }^{2}$, como «to find out what other animals want, it is not enough merely to study them. We need to talk with them» (p. 231), esto es, «having conversations with animals» (p. 231). No lo es menos que Meijer se lamente de que «many laws and regulations affect the lives of other animals, even though they are not consulted» (p. 223), al asumirse que no pueden hablar. Pero según Meijer, «it has become clearer that this is simply not true, and we should not ignore them any longer» (p. 223), llegando a sugerir incluso que "we can operate on the basis of their languages [...] to explain thing to them» (p. 224). También señala que «it is too early to reach final conclusions about animal languages and to give a full definition of animal language» (p. 216) porque «humans in isolation should never reach such a conclusion» (p. 216). No sabemos cómo los animales podrían participar en la discusión de las leyes que les afectan, qué es lenguaje o cómo podríamos 'hablar' con ellos.

Según Meijer, rechazar la existencia de lenguaje en animales revela antropocentrismo («the notion that humans are the centre of existence»; p. 7), de modo que esa posibilidad suele abordarse «as if there were a huge gap between our forms of communication and theirs, and as if human language were more elevated, something that animals will never be able to achieve» (p. 6), menospreciando así las formas de expresión no humanas como inferiores (p. 231). Meijer se adhiere a una acusación usual entre etólogos, según la cual sostener que el lenguaje es únicamente humano implica un antropocentrismo que acentúa la diferencia entre humanos y animales (cfr. 
Savage-Rumbaugh et alii 1998: caps. 2-4; Segerdahl et alii 2005); por tanto, la posesión del lenguaje por animales sería rechazada por razones ideológicas, no científicas.

Aunque Meijer considere que hay que 'hablar' con los animales, estos no pueden hacerse oír; por ello, al propugnar el igualitarismo entre animales y humanos, referencias como las referidas en el párrafo previo proyectan a los animales como acreedores de muchos derechos humanos. A mi juicio, no es antropocéntrico, sino de justicia, proponer que otros seres merecen el mismo respeto y protección que los humanos creemos merecer. Sin embargo, Meijer (al igual que los restantes autores que comparten esa acusación) incurre en el defecto que desea combatir, el antropocentrismo, pues evaluar los rasgos de otros seres con parámetros ajenos a ellos convierte lo humano en medida del reino animal: al sostener lenguaje en animales, Meijer los acerca a parámetros específicamente humanos. Por ello señalaba Budiansky (1998: 18) que «en su batalla contra el antropocentrismo, han adoptado la postura más antropocéntrica posible» (cfr. Deacon 1997: 52-53 y Tomasello 2003: 9-10 para reflexiones semejantes). Apuntar las diferencias entre especies no supone más que considerar la evolución de cada una como divergente con respecto a las demás: «humans are indeed unique - no more so than every other species, but not less so either» (Anderson 2004: 324). Por tanto, cada sistema comunicativo es único.

Por ejemplo, basándose en Slobodchikoff (2012), Meijer señala que aunque la estructura del canto de los colibríes es bien conocida, «we know little about that meaning though» (p. 170). Pero eso no le impide 'traducir' alegremente las señales de los colibríes a oraciones: «it is likely that the messages reflect the bird's intentions, ranging from 'clear off' or 'come on if you dare' to 'I know where you live» (p. 170). Esta estrategia aplica el antropocentrismo que Meijer al tiempo denuncia. Si en vez de efectuar un análisis autónomo de los sistemas comunicativos animales los relacionamos con un sistema ajeno a ellos como el lenguaje, «we run the risk of underestimating the animals and thereby missing what is interesting, complex, and significant about these creatures' abilities and actions in their own right» (Anderson 2004: 40).

De nuevo, la autora incurre en otra contradicción: mientras por un lado afirma insistentemente que los códigos animales poseen todo tipo de rasgos lingüísticos, por otro sostiene, al respecto de los primates, que «we need to develop research based on their [primates] view of the world» (p. 31). Si es así, no se entiende por qué atribuye lenguaje a esos seres, pues es obvio que los procesos semiótico-representacionales que construyen su realidad no dependen del lenguaje.

Hay también muestras de antropocentrismo fuera del ámbito comunicativo. Según Meijer, algunos animales hacen funerales y ritos funerarios: «crows (like ravens, magpies and some other birds) hold funerals when a member of their group dies» (p. 43), o «crows, elephants and other animals have mourning rituals» (p. 105). Incluso afirma que «it would be premature to deny that they have an understanding of death» (p. 105) o que los elefantes muestran un «abstract understanding of death» (p. 39; cfr. también p. 208). Pero una cosa es que muchos animales muestren tristeza inequívoca ante la muerte de un congénere (algo bien atestiguado) y otra muy diferente es sostener que los animales tienen ritos funerarios, o que entienden la muerte de manera abstracta, una conducta hasta el momento únicamente atestiguada en humanos y que, de nuevo, sugiere antropocentrismo al aplicarse a animales. 


\section{TERGIVERSACIONES DE FUENTES}

En el caso discutido sobre los supuestos funerales animales, y usando una estrategia recurrente, Meijer tergiversa el contenido de una referencia utilizada como fuente, al sugerir que tal referencia afirma algo que en realidad no hace. En concreto, Meijer cita a King (2013) como supuesto respaldo a su afirmación de que sería prematuro negar que los animales entienden la muerte de modo abstracto (nota 9, p. 251). En realidad, King (2013) señala algo muy diferente de lo que Meijer dice que dice: según King, los animales experimentan dolor por la muerte de congéneres, pero escribe que «do some animals grasp death's finality or even have a mental concept of death? We simply don't know. No evidence suggests that any nonhuman animal anticipates death in the way we humans do» (King 2013: 67).

Este caso no es el único donde Meijer tergiversa las fuentes; por ejemplo, afirma que «recent research into giraffes has revealed similar mourning rituals» (p. 208), apoyándose en Baotic et alii (2015) (nota 39, p. 263). Para nuestro asombro, Baotic et alii (2015) no aluden en ningún momento a las conductas mortuorias de las jirafas. Este trabajo estudia las vocalizaciones de esos animales, señalando que, en las 947 horas de grabaciones hechas a jirafas de tres zoos, «we found no evidence for giraffe infrasonic communication in our data set even though it is widely assumed that giraffes communicate in this manner» (Baotic et alii 2015: 8). Además, «we [...] documented a sound that has never been structurally described in the scientific literature before» (Baotic et alii 2015: 5), las vocalizaciones de tarareo (Baotic et alii 2015: 5), emitidas principalmente por la noche, señalando que «we are unable to make any statement about the context-specific use, or the potential active or passive communicative role of humming» (Baotic et alii 2015: 8). Por tanto, este trabajo no alude a ningún tipo de ritual mortuorio en jirafas.

En otro ejemplo de tergiversación, Meijer sostiene, basándose supuestamente en Smith \& Johnson (2012) (nota 27, pp. 252-253), que «chickens make use of sight, touch and scent to communicate about present, past and future» (p. 124). Esta afirmación presupone la propiedad de desplazamiento, por la cual «aquello a que se refiere la comunicación puede estar alejado en tiempo y espacio del momento y lugar en que se establece la comunicación» (Hockett 1958: 560), y es impactante, porque no hay evidencia de ella en la comunicación animal (exceptuando la danza de las abejas, donde siempre hay desplazamiento espacial), que se restringe a «the here and now» (Hauser et alii 2002: 1576). Sin embargo, en ningún momento señalan Smith \& Johnson (2012) que las aves de corral, como los pollos, comuniquen sobre el pasado y el futuro. Por un lado, muestran que esas aves tienen varias señales referenciales sobre predación y comida, emitidas en presencia de los elementos a que aluden. Por otro, sostienen que disponen de capacidades cognitivas complejas, como inferencia transitiva y aprendizaje social, de manera que «hens observing the interactions of a known status individual with an unknown individual are able to infer their own status relative to the unknown individual and to respond appropriately in future interactions» (Smith \& Johnson 2012: 81), de manera dominante o bien sumisa. Pero esas inferencias son puramente mentales, no se comunican, por lo que de nuevo no cabe el desplazamiento.

Un caso especialmente relevante de la manipulación de una fuente es cómo refleja Meijer el fascinante trabajo de Irene Pepperberg con el loro gris africano Alex, que reveló capacidades cognitivas muy destacadas en esta especie (Pepperberg 1999). Según Meijer, Pepperberg «wanted to investigate if parrots were capable of learning language» (p. 18), mostrando que "parrots can develop language» (p. 22). Lo cierto es que la intención de Pepperberg no fue enseñar el lenguaje a Alex, sino usarlo como una mera herramienta para revelar sus capacidades cognitivas (formación 
de conceptos, etc.). La propia Pepperberg (1999: 34-35) escribe unas palabras que desacreditan la interpretación de Meijer:

researchers who train animals to use a human-based code in order to study nonhuman cognition often lose sight of the code as an investigative tool and argue instead about the extent to which the code is equivalent to human language [...]; that is, they compare human and nonhuman linguistic rather than cognitive abilities. Thus data and discussions about relative cognitive capacities are lost amidst open-ended debates about the extent to which a particular animal has acquired 'language' or how well particular studies or procedures demonstrate linguistic competence [...] In all the material to follow, we must remember that the power of interspecies communication is best exploited by focusing on how an animal's use of a vocal code facilitates investigations of its cognitive abilities, rather than on what the animal's use of the code itself implies about such abilities.

La interpretación de Anderson (2004: 33) es muy diferente de la muy desafortunada de Meijer: «Pepperberg has no illusions that Alex is learning English», sino que «she is interested in exploring the parrot's cognitive abilities, and in that endeavour, (some aspects of) language can serve as a tool, rather than necessarily as the object of inquiry».

Meijer también señala que Alex conocía «how sentence structure works» (p. 19). Esto es también inexacto: Pepperberg (1999: 140) refiere que el trabajo con mamíferos marinos «specifically tested whether they could respond on the basis of word order, but Alex's task did not: his task emphasized semantic comprehension», por lo que «word order was intentionally removed as a cue for Alex» (p. 140).

Pero tras sostener insistentemente que Pepperberg «wanted to investigate if parrots were capable of learning language» (p. 18), en la misma página señala que «Pepperberg is not arguing that Alex can speak English, simply that he uses words and concepts and therefore shows understanding and intelligence» (p. 22). ¿En qué quedamos, pues? Meijer tiene la curiosa habilidad de afirmar una cosa y la contraria. Otro de esos vaivenes consiste en sostener por un lado insistentemente que diferentes sistemas animales tienen gramática («the grammar of birds», p. 186; «the languages of whales, octopuses, bees and many birds have a grammar», p. 1), pero por otro que «research does not yet give us an unambiguous answer to the question of whether animal languages have grammar» (p. 182), algo que a su juicio depende de cómo se defina ésta. Por cierto, la definición que ofrece de ella es cuando menos curiosa: «the body of rules and principles for speaking and writing a language» (p. 172).

Como ultimo ejemplo de tergiversación, Meijer afirma, apoyándose en Mather (2008) (nota 1, p. 256), que «cephalopods [...] are believed, on the basis of brain and behavioural research into memory and the ability to learn, to have a consciousness» (p. 163). Pero lo que dice Mather (2008: 37) es que los cefalópodos tienen una forma de "primary consciousness», algo bien diferente. En realidad, existen varios tipos de conciencia (Van Gulick 2018), de la que la primaria es el tipo básico. Edelman (2004: 9) expone la diferencia entre la conciencia primaria y la de orden superior (cfr. también Edelman et alii 2011):

primary consciousness is the state of being mentally aware of things in the world, of having mental images in the present. It is possessed not only by humans but also by animals lacking semantic or linguistic capabilities whose brain organization is nevertheless similar to ours. Primary consciousness is not accompanied by any sense of a socially defined self with a concept of a past or a future. It exists primarily in the remembered present. In contrast, higher-order consciousness involves the ability to be conscious of being conscious, and it allows the recognition by a thinking subject of his or her own acts and affections. It is accompanied by the ability in the waking state explicitly to recreate past episodes and to form future intentions. 
Por otro lado, en ocasiones Meijer justifica sus afirmaciones aduciendo referencias sin relación alguna con lo afirmado. Por ejemplo, señala (p. 89) que en los años 60 Hockett formuló su bien conocido sistema de rasgos (en realidad, fueron formulados ya en Hockett 1958); a pesar de ello, la referencia aducida al respecto es Hockett (1942) (cfr. nota 75, p. 250), trabajo que no trata tales rasgos (formulados 16 años después) sino que "delimits the field of descriptive phonology, formulates a system thereof, and briefly points out some of its implications" (Hockett 1942: 3). Otro ejemplo no menos chocante es que Meijer señala que Chomsky es uno de los principales críticos del conductismo (p. 136), pero sustenta esta afirmación (nota 3, p. 254) en Chomsky (1957) en lugar del trabajo clave al respecto, Chomsky (1959), que no cita.

\section{LA CONCEPCIÓN SEMIÓTICA Y LINGÜÍSTICA DE MEIJER}

El conocimiento que Meijer muestra de aspectos semióticos es en ocasiones muy pobre. A su juicio, la comunicación de la lura del Caribe (Sepioteuthis sepioidea) «satisfies a number of criteria that we consider specific to human language» (p. 164), afirmando que «the colours appear, for example, to be able to refer to aspects of the outside world» (p. 164). Meijer parece desconocer que poder aludir a aspectos del mundo supone la propiedad de semanticidad (Hockett 1958), por la cual «los elementos de un sistema de comunicación tienen denotaciones - es decir, tienen lazos asociativos con cosas y situaciones, o con tipos de cosas y situaciones, del entorno de quienes los emplean» (Hockett 1958: 557-558). Aunque el lenguaje posee semanticidad, este rasgo no es exclusivo de él, frente a lo que sostiene Meijer, sino propio también no solo de algunos sistemas comunicativos animales sino también de múltiples sistemas semióticos humanos: sin semanticidad no habría signos.

Meijer también indica que los rasgos 7 y 8 de Hockett (semanticidad y arbitrariedad) «are about meaning» (p. 89), pero de nuevo comete el error de vincularlos solo con los signos lingüísticos, al señalar que «they concern semantics, the meaning of a word» (p. 89) (quizás esto derive de su idea de que todo es lenguaje, esto es, de que los animales poseen lenguaje). Al primero de ellos ya aludí antes; el segundo, es un criterio básico en la clasificación de los códigos semióticos de cualquier ámbito (Simone 1990: 33 y ss.), plasmándose por doquier fuera del lenguaje.

Dada la tesis del libro de que la comunicación animal es lenguaje, Meijer le atribuye todo tipo de propiedades o unidades lingüísticas. Por ejemplo, palabras: la autora considera de modo simplista que el hecho de que un animal entienda o emita palabras muestra que las posee. Esta idea, en sintonía con propuestas previas que atribuyen esas unidades (o equivalentes funcionales de ellas) a animales (cfr. Seyfarth 1980a, 1980b sobre las llamadas de alarma de los cercopitecos), revela deficiencias en la comprensión de qué son y cómo se adquieren las palabras, que parece apuntar a un proceso muy simple «at least to scholars who are not directly engaged in its study» (Bloom 2000: 3).

Por ejemplo, puesto que Kosik, elefante asiático de un zoo de Corea del Sur, podía pronunciar palabras, según Meijer «he [Kosik] speaks to her [una hembra] in elephant language, and continues to use human language with the people around him» (p. 37). Stoeger et alii (2012) muestran que Kosik tiene capacidad de imitación vocal, descrita en pájaros oscinos y algunos mamíferos (cfr. Janik \& Slater 1997), usando formantes propios del coreano. Según Stoeger et alii (2012: 2144), Kosik emite cinco palabras coreanas: 'annyong' (hola), 'anja' (siéntate), 'aniya' (no), 'nuo' (túmbate) y 'choah' (bien), siendo el porcentaje de similitud en las vocales un 67\% y el de las 
consonantes un 21\%. Pero Meijer deja de lado lo más relevante del estudio, que desde luego no es que Kosik 'use el lenguaje': este elefante utiliza la trompa para producir sonidos imitados, colocándola dentro de la boca, por lo que puede modular el tracto vocal, algo desconocido en elefantes asiáticos y que Kosik no adopta cuando produce sonidos de elefante. Por tanto, este caso muestra que los elefantes pueden superar limitaciones morfológicas aumentando el tracto vocal con la trompa (Stoeger et alii 2012: 2145), lo cual representa «a wholly novel method of vocal production and formant control in this or any other species» (Stoeger et alii 2012: 2144). El resultado es muy importante porque el estudio comparativo de la imitación vocal es fundamental para entender los mecanismos biológicos de un rasgo clave del habla y cómo esta pudo evolucionar (Lattenkamp \& Vernes 2018), pero no revela que Kosik posea palabras o lenguaje. Debe tenerse en cuenta que aunque el aprendizaje de palabras en el proceso de adquisición del lenguaje precisa una capacidad de imitación vocal (o manual en las lenguas de signos), la capacidad de imitación vocal en animales no humanos está disociada de la semanticidad, por lo que no conlleva aprendizaje 'léxico' en el sentido fuerte del término; de hecho, las señales de los cantos de estos imitadores vocales en sus señales carecen de semanticidad o referencialidad ( $c f r$. infra).

Meijer también aduce los estudios sobre dos perros collies (pp. 97-99), Rico (Kaminski et alii 2004) y Chaser (Pilley \& Reid 2011), que adquirieron «a large vocabulary» de palabras (p. 99), llegando a 1.022 en Chaser. Cuando los dueños pronunciaban la palabra para un objeto, los perros cobraban ese objeto y se lo llevaban. Ambos casos son sorprendentes, al mostrar que los perros pueden sobrepasar grandemente el número de asociaciones que los primates establecen en laboratorio. Pero sostener que los perros han adquirido palabras desde la óptica de la comprensión (algo asumido por los estudios citados y por Meijer) supone malentender qué son las palabras (cfr. Longa \& López Rivera 2005), e ignorar lo realmente importante, las vastísimas capacidades de categorización y memoria de esos animales.

En realidad, Meijer identifica las palabras con meras etiquetas para objetos. Esto se asienta en la concepción popular de palabra (criticada por Bloom 2000 o Tomasello 2003), según la que su adquisición solo supone establecer un trazado directo entre nombres y referentes. Pero las palabras tienen propiedades bien diferentes.

Sin duda, las palabras evocan en Rico y Chaser una representación mental de los referentes correspondientes (en caso contrario, no podrían identificar objetos), pero las palabras producen en ellos siempre la misma respuesta instrumental, cobrar el objeto. Por ello, las palabras escuchadas por los perros son etiquetas de conceptos-objetos referidas a aspectos concretos y vinculadas con el aquí y ahora (en cuanto escuchan la palabra, cobran el objeto). Por el contrario, las palabras carecen de cualquier vínculo instrumental (podemos usar 'pelota' en contextos muy diferentes), de modo que no se basan en un trazado directo entre palabra y objeto. De hecho, las palabras pueden caracterizar conceptos de objetos reales como abstractos o irreales, sin referente en el mundo. Ya Saussure (1916) rechazó que una lengua se redujera a una mera nomenclatura (listas de términos referidas a listas de cosas). Lo señalado sugiere una diferencia fundamental entre las palabras y las etiquetas que estas son para los perros (Bickerton 1990: 50-51; Györi 1995: 120; Longa \& López Rivera 2005: 308): las palabras liberan a los conceptos de su dependencia con respecto a la percepción sensorial, permitiendo así concebir elementos sin referente en el mundo. Además, aprendemos asociando palabras con palabras (Quine 1960: 31 y ss.): si nos cuentan los rasgos que definen un concepto, podemos entablar la relación entre una palabra y el concepto asociado antes de saber si existe un objeto real para ese concepto. Sin embargo, lo que escuchan 
los perros produce representaciones de objetos directamente ligadas a la percepción ${ }^{3}$, siendo así etiquetas para objetos reales, vinculados con el aquí y ahora, igual que el resto de señales animales (Hauser 2000: 267; Hauser et alii 2002: 1576). Notemos que también las palabras pronunciadas por Kosik se vinculan con instrucciones concretas en un contexto de adiestramiento. En resumen, «the terms of human languages are radically different from those of animal symbolic systems. The latter appear to be associated with mind-independent events, while even the simplest human terms violate this condition» (Chomsky 2017: 201).

Para resumir, la diferencia entre las palabras que escuchan esos perros y las palabras es la que existe entre un conocimiento directamente ligado a la experiencia en animales frente a uno posible en ausencia de ella en nosotros. Los perros no adquirieron palabras sino etiquetas. Por otro lado, a pesar de la diferenciación entre léxico y sintaxis, el primero configura en gran medida a la segunda, dadas las propiedades de subcategorización de las piezas léxicas, que determinan las combinaciones de palabras.

Meijer incluso va más allá, sosteniendo tipos concretos de palabras en algunos animales; por ejemplo, en la lura del Caribe, cuya comunicación fue estudiada por Moynihan (1985). Esta obra cataloga muchos componentes de la piel de este animal con papel comunicativo, aislados o combinados. Sin embargo, ese trabajo pionero adolece de un grave problema, cual es la conclusión (que Meijer adopta) de que la comunicación de las luras mediante la piel se asemeja al lenguaje, al suponer que esos componentes podrían equivaler a verbos, sustantivos, adjetivos o adverbios.

También sostiene Meijer que los perritos de las praderas «use verbs, nouns and adverbs in meaningful constructions» (p. 61) basándose en los estudios de Slobodchikoff sobre la comunicación de esa especie (Slobodchikoff 2012; Slobodchikoff et alii 1991, 2009). La razón consiste en que los perritos emiten llamadas de alarma que no solo indican cuatro amenazas concretas (perros, halcones, coyotes y humanos) sino incluso propiedades de esas amenazas. Por ejemplo, Slobodchikoff et alii (2009) expusieron a tres mujeres de la misma estatura, vistiendo camiseta azul, verde y amarilla respectivamente, a una colonia de perritos. El análisis acústico de las llamadas reveló que las referidas a las mujeres con camiseta azul y amarilla diferían entre sí, pero esta última no difería de la llamada sobre la mujer con camiseta verde. Ya que la respuesta de escape fue la normal, las diferencias acústicas reflejan propiedades sobre rasgos de las mujeres en vez de sobre cómo escapar de la amenaza ${ }^{4}$. Por ello, «these findings suggest that prairie dogs have the cognitive capacity to recognize individual differences in the appearance of possible predators and are able to incorporate information about these differences in their vocalizations» (Slobodchikoff et alii 2009: 438). Esta indicación de rasgos de la amenaza (compartida por otras especies, como el carbonero de capucha negra; cfr. Templeton et alii 2005) muestra que los perritos pueden establecer categorizaciones muy precisas y comunicarlas, pero poco tiene que ver con que esos animales posean verbos o sustantivos, afirmación que se antoja un mero trasvase antropocéntrico de las propiedades de las llamadas.

Lo más chocante es que, según Meijer, estudiar casos como este «teaches us not only about the communication systems of other animals, but also about how they experience and view the world» (p. 59). Si es así, ¿qué aporta defender que hay clases de palabras en vez de sostener que pueden establecer y comunicar categorizaciones muy precisas?

La concepción de Meijer sobre la sintaxis es no menos pobre. Basándose en Zuberbühler (2002), afirma que «the alarm call of the Campbell's monkey has syntax: elements are connected as in the structure of a sentence» (p. 62). Estos monos africanos tienen dos gritos de alarma emitidos ante 
leopardos o águilas y, al tiempo, tienen también una llamada breve que puede preceder al grito de alarma (nunca se emite aislada) si el peligro no es inminente y que, a diferencia del grito aislado, no provoca la huida (posteriormente, Ouattara et alii 2009 ampliaron el elenco de gritos). Por tanto, esa combinación supondría una regla sintáctica que altera el significado del mensaje resultante. Sin embargo, aunque el interés de ese sistema es muy grande, difícilmente es asimilable a la sintaxis, si esta se considera seriamente ${ }^{5}$. Esta se define por criterios jerárquicos (verticales), no horizontales (lineales), que son los que rigen en este caso (cfr. Bickerton 1990: 83-92, y la crítica de Bickerton 1998 a visiones reduccionistas semejantes a la tratada aquí). Mientras la sintaxis se caracteriza por la jerarquía y la recursión, la regla mencionada carece de ambas propiedades, siendo una concatenación puramente lineal, muy bien documentada por otro lado en animales (Hornstein 2009: 112). Como señala Anderson (2004: 298), «if one believes that syntax is simply a matter of putting words (or signs) together one after another, the burden of proof is not huge».

Por otro lado, Meijer trata repetidas veces el canto de varias especies, como ballenas o pájaros oscinos (para otros casos de canto, cfr. Longa 2013), señalando por ejemplo que los cantos de ballenas «involve grammar» (p. 177) o aludiendo a «the grammar of birds» (p. 168). En efecto, uno de los mayores hallazgos sobre la comunicación animal fue descubrir que esos cantos tienen una estructura jerárquico-combinatoria semejante a la del lenguaje, pues las señales se forman mediante partes constituyentes y existen varios niveles jerárquicos, que en las ballenas pueden llegar a cinco (sobre el canto de ballenas, cfr. Payne \& McVay 1971; Suzuki et alii 2006; Cholewiak et alii 2013; sobre las propiedades del canto de pájaros, cfr. Berwick et alii 2011, 2012; Bolhuis et alii 2010; Bolhuis \& Everaert eds. 2013; Marler \& Slabbekoorn eds. 2004). Pero aunque los cantos tienen estructura sintáctica, Meijer omite que «birdsong cannot be directly compared with the syntactic complexity of human language, principally because it has neither semantics nor lexicon" (Berwick et alii 2011: 114). Esto es, la sintaxis de los cantos no se aplica a elementos con significado sino a segmentos fónicos, y diferentes combinatorias no producen nuevos significados (Longa 2015). Por ello, Meijer está errada cuando afirma que en los cantos «there is room for meaningful additions that make new sentences» (p. 169).

Además, Meijer indica que la investigación del canto de los pájaros «has yielded little information about the meaning of bird languages» (p. 91). Esto es inexacto, porque de entre todas las especies que tienen cantos, el de los pájaros es el mejor conocido, teniendo dos funciones básicas: reclamo de pareja y defensa del territorio. Además, existe consenso en que esos cantos carecen de contenido referencial o 'semántica' (Marler 1998: 11), teniendo un contenido puramente emotivo (Anderson 2004: 141; Berwick et alii 2011: 118; Hauser 1996: 476; Slater 1999: 179). Aunque los demás sistemas de canto no son tan conocidos, todo apunta a la misma ausencia de referencialidad en ellos (por ejemplo, Hurford 2012: 96 señala que el contenido informativo de los cantos de ballenas es «very low»).

Por otro lado, de nuevo Meijer asevera una cosa y la contraria: señala que la recursión «has certainly been demonstrated in the language of various birds» (p. 91) o que los estorninos «are capable of understanding new recursive additions to their language» (p. 169), mientras que en la misma p. 169 indica que «the answer [a si existe recursión en pájaros] is disputed».

Más sorprendente es aún que afirme que «it is quite posible that this [recursión] also applies to the language of elephants» (p. 91), sin indicar fuente alguna. En efecto, «recursion is a common behavior used by elephants» (English et alii 2014: 551), pero en un sentido completamente diferente al de Meijer, ni siquiera vinculado con la comunicación: «recursion by herbivores is the 
repeated use of the same site or plants» (English et alii 2014: 551), implicando «returns to previously visited areas» (Berger-Tal \& Bar-David 2015: 1).

Es cierto que Hauser et alii (2002: 1578) sugieren que la recursión podría haber evolucionado para resolver problemas computacionales como la navegación, y si así fuera, algunos animales podrían tener esta propiedad. Pero para afirmar esto último, Meijer debería mostrar que la estrategia de recursión en elefantes tiene los tres rasgos de la recursión en el lenguaje (Hauser \& Watumull 2017; Watumull et alii 2014): computabilidad (enumeración explícita de modelos), inducción matemática (que permite la generación ilimitada) y especialmente, definición por inducción (de la que resulta la estructura jerárquica). Como escriben Watumull et alii (2014: 5):

animal navigation by path integration (dead reckoning) requires the carrying forward of vector values: displacements are summed to plot a path. However, to demonstrate equivalence with linguistic recursion, this process would need its outputs to be not only returned as inputs but also represented hierarchically. Summing vectors just generates another vector (in a tail recursive process), not a hierarchical structure over which can be defined complex relations (e.g., syntactic, semantic, phonological, etc.).

Tal vez Meijer haya visto que el término de recursión se aplica a los elefantes, creyendo que aludía a la recursión lingüística (lo cual implicaría que no ha leído las referencias relevantes). En todo caso, su afirmación está vacía de contenido.

\section{ERRORES GRAVES}

Lo tratado hasta ahora, que no es poco, no es lo más negativo del libro. A ello hay que añadir errores graves, de los que ofrezco varios ejemplos muy destacados. El primero alude a la noción de innato, que Meijer acepta. Basándose en Konrad Lorenz (muy cuestionado, sin embargo, en su adopción de esa noción; cfr. Longa \& Lorenzo 2019; Lorenzo \& Longa 2018: cap. 6), Meijer señala que «reacting to call-notes is innate for many animals: they do it automatically and do not need to learn anything in order to do it» (p. 41), y que «a number of forms of expression are innate, such as facial expressions in humans or the call for the mother in ducks» (p. 191).

Sostener que el reconocimiento de las llamadas maternas de patos es innato revela un profundo desconocimiento, pues precisamente ese caso es un ejemplo paradigmático de un rasgo antaño considerado innato pero que dejó de ser concebido como tal hace ya decenios, y que cualquier manual introductorio de psicobiología del desarrollo expone. Gottlieb (1971) demostró que el reconocimiento de esas llamadas no es un rasgo innato, al requerirse la experiencia prenatal del embrión en forma de aprendizaje no obvio (cfr. Gottlieb 1992, 1997 sobre las grandes repercusiones de ese hallazgo, y Lorenzo \& Longa 2018: cap. 6 para una síntesis).

Previamente a Gottlieb se conocía que varias aves (ánade real, pato joyuyo, ánade rabudo o pollo doméstico) pueden identificar la llamada materna sin exposición previa a ella, por lo que este rasgo se tomaba como instintivo. Aunque Gottlieb asumía que la ausencia de estímulo materno veta que la experiencia contribuya en forma de aprendizaje franco (generalización de estímulos), eso no significaba que la experiencia fuera irrelevante para el reconocimiento (Gottlieb 1997: 19). Por ello, empezó a analizar factores no obvios, descubriendo que los embriones de pato empiezan a emitir vocalizaciones antes de la eclosión del huevo, y que auto-escuchar las propias vocalizaciones en fase embrionaria es clave para que el pato pueda reconocer la llamada materna incluso sin haber 
escuchado esta y aunque ambas vocalizaciones son muy diferentes. Por tanto, el reconocimiento de la llamada no es innata ni está prefijada en los genes.

El trabajo de Gottlieb muestra que la conducta en el recién nacido no es un instinto y también que la experiencia no empieza tras el nacimiento: «the absence of frank learning or practice does not mean that experience does not play a role in the ontogeny of instinctive behavior» (Gottlieb 1997: 57). Por ello, el desarrollo del reconocimiento de la llamada materna tiene una naturaleza intrincada, porque

not only must the duckling experience the vocalizations as an embryo (the experience is ineffective after hatching), the embryo must experience embryonic vocalizations. That is, the embryonic vocalizations change after hatching and no longer contain the proper ingredients to tune the embryo to the maternal call (Gottlieb 1992: 169-170).

En resumen, al afirmar que el reconocimiento de las llamadas de pato es un rasgo innato, Meijer revela un profundo desconocimiento y falta de actualización.

No sorprende menos leer que, según Chomsky, el lenguaje «is not intended primarily for communication but to understand the world better» (nota 6, p. 256). Me temo que Chomsky no concordaría en absoluto con esta interpretación, que reduce el lenguaje a un mero papel externista.

Por otro lado, Meijer afirma varias veces que las llamadas de alarma revelan altruismo recíproco, de modo que «the caller draws attention to itself with the aim of helping others» (p. 76). Ciertamente, bastantes etólogos suscribirían el altruismo de las señales, pero Meijer silencia que esa visión no es unánime, quizás por desconocimiento o tal vez porque no encaja con su propio marco preestablecido sobre los animales.

Una vez que se empezó a constatar la comunicación compleja de muchas especies, esta se comenzó a analizar suponiendo que existe transmisión de información (según el modelo humano de transmisión de significado) y que esa transmisión es altruista, emitida para beneficiar a los congéneres. Pero esa visión fue desafiada por Dawkins \& Krebs (1978) (perspectiva basada en Dawkins 1976), que explican el aparente altruismo comunicativo como una manipulación para satisfacer el propio interés egoísta. Este marco fue llevado a su máxima expresión por el modelo de 'evaluación-gestión' de Owings \& Morton (1998), según el cual un animal no comunica para ayudar al grupo sino para ayudarse a sí mismo. La relación entre evaluación (por el receptor) y gestión (por el emisor) se resuelve en términos de un análisis de costes y beneficios: solo si comunicar beneficia al emisor este emitirá una señal, y el receptor solo actuará si obtiene un beneficio. Desde esta óptica, por ejemplo, el grito de alarma de un mono ante un depredador se emite egoístamente, no para ayudar al grupo: si el mono que divisa al depredador escapa sin avisar, ser el único mono que repentinamente se mueve para escapar lo convertiría en presa prioritaria; pero si emite una señal que provoca una estampida del resto de monos, todos ellos pasan a ser presas potenciales, con lo que el depredador desvía su atención del mono que emitió la llamada. Así, los beneficios superan a los costes: quien emite el grito sobrevive (gestión) y quien escapa también sobrevive (evaluación).

La polémica entre altruismo y egoísmo sigue vigente: Rendall et alii (2009: 238) sostienen que el marco altruista es incoherente, al erigirse sobre una falsa analogía con el lenguaje, siendo «both teleological and circular in using constructs developed for one recently evolved and possibly highly derived system of communication (language) to model processes involved in scores of other simpler and phylogenetically older systems in other species». Por ello, el marco informacional 
(altruista) debería reemplazarse por otro basado en los intereses de emisor y receptor, según el cual la señal no pretende informar a los congéneres sino influir en ellos. Sin embargo, Seyfarth et alii (2010) sostienen que el marco informacional es fundamental para analizar la comunicación animal (cfr. Owren et alii 2010, que discute las implicaciones de ambas visiones). Por tanto, Meijer presenta una perspectiva como la única existente y decide ignorar la otra.

Sin duda, el error más grave del libro, que descalifica a su autora, es sostener que FOXP2 es el gen del lenguaje. Según Meijer, «in all vertebrates - including long-extinct species such as Neanderthals - the FOXP2 gene, more commonly known as the language gene, has been found», sosteniendo que «it is not only responsible for language, but is also linked to forms of learning» (p. 87). Considerar FOXP2 como el gen del lenguaje, o el gen responsable del lenguaje, es, sencillamente, un disparate mayúsculo (cfr. Longa 2006).

Lai et alii (2001), quienes descubrieron la vinculación de este gen con el lenguaje, mostraron una correlación inequívoca entre la versión mutada del gen en algunos miembros de la familia KE y diferentes trastornos (cognitivos y lingüísticos), pero a partir de tal correlación no se puede deducir la relación directa entre gen y rasgo (FOXP2 y lenguaje), aspecto que es de primer curso de biología pero que Meijer ignora.

Por un lado, Meijer revela desconocer el papel de los genes al considerar que estos pueden contener un rasgo como el lenguaje: «genes store information coding for the amino acid sequences of proteins; that is all. They do not code for parts of the nervous system and they certainly do not code for particular behavior patterns» (Bateson 2001: 157). Por otro, la relación entre genes y rasgos no puede ser directa, sino enormemente indirecta, no solo para los rasgos cognitivos sino incluso para los fisiológicos ( $c f r$. Blumberg 2005; Moore 2001; Moss 2003). Como escriben Fisher \& Vernes (2015: 291), «it is not possible for a gene to specify an individual cognitive process», pues entre los genes y los rasgos existe un trazado muy complejo debido a la gran cantidad de niveles y factores existentes (Johnston \& Edwards 2002 muestran esa complejidad al postular 13 diferentes niveles en el trazado entre genes y conducta), sin todos los cuales los genes «are among the most impotent and useless materials imaginable» (West-Eberhard 2003: 93; cfr. Benítez Burraco \& Longa 2011; Longa \& Lorenzo 2012 sobre las razones por las que el vínculo directo genes-rasgos es una entelequia).

La adopción por parte de Meijer del supuesto vínculo directo entre genes y rasgos, remite a la genética clásica (mendeliana-morganiana), concebida como el análisis de unidades discretas actuando sobre rasgos fenotípicos concretos, y considera al gen un agente causal simple. Esto es lo que conduce a creer que un gen puede ser el responsable directo y único de un fenotipo (como el lenguaje). Esa concepción está completamente errada, como señalan Jablonka \& Lamb (2005: 5):

although many psychiatrists, biochemists, and other scientists who are not geneticists (yet express themselves with remarkable facility on genetic issues) still use the language of genes as simple causal agents, and promise their audience rapid solutions to all sorts of problems, they are no more than propagandists whose knowledge or motives must be suspect. The geneticists themselves now think and talk (most of the time) in terms of genetic networks composed of tens or hundreds of genes and gene products, which interact with each other and together affect the development of a particular trait.

Que exista una correlación entre una secuencia de ADN y la presencia de un rasgo no supone una causación directa del rasgo por parte de esa secuencia: descubrir que una secuencia de ADN mutada afecta a un rasgo no presupone que la secuencia funcionalmente correcta origine por sí 
misma ese rasgo: «a disconnected wire can cause a car to break down, but this does not mean that the wire by itself is responsible for making the car move» (Bateson 2001: 157). Ningún gen puede ser el responsable del lenguaje ni de cualquier rasgo y aún menos en este caso, dado que FOXP2 es un gen de tipo regulador. Por ello, «FOPX2 cannot be regarded as 'the' gene 'for' language, since it is only one of many that have to be functioning properly to permit its normal expression» (Bolhuis et alii 2014: 3).

\section{CONCLUSIÓN}

Para finalizar, el libro de Meijer comete la falacia de intentar vincular la comunicación compleja en animales con su supuesta posesión de lenguaje. Esta estrategia no solo es innecesaria, sino que minusvalora los sistemas animales, al pasarlos por un filtro que les es completamente ajeno. Pero más allá de ello, el libro es calamitoso: tergiversaciones de autores, contradicciones, desconocimiento de aspectos semióticos y lingüísticos básicos, errores al aducir referencias, errores conceptuales muy graves, etc. No se entiende cómo la autora ha escrito un libro así, y, sobre todo, tampoco se entiende cómo la editorial John Murray (y antes la editorial holandesa ISVW) lo ha publicado.

\section{Bibliografía}

Anderson, S. R. (2004): Doctor Dolittle's Delusion. Animals and the Uniqueness of Human Language. New Haven: Yale University Press.

BAOTIC, A., F. SiCKS \& A. S. STOEGER (2015): «Nocturnal 'humming' vocalizations: Adding a piece to the puzzle of giraffe vocal communication», BMC Research Notes 8: 425, pp. 1-11. https://doi.org/10.1186/s13104-015-1394-3

BATESON, P. (2001): «Behavioral development and Darwinian evolution», en S. Oyama, P. E. Griffiths \& R. D. Gray (eds.): Cycles of Contingencies. Developmental Systems and Evolution. Cambridge (MA): MIT Press, pp. 149-166.

Benítez Burraco, A. \& V. M. LONGA (2011): «El papel del ADN fósil en Paleoantropología: FOXP2, Neandertales y lenguaje», Zephyrus. Revista de Prehistoria y Arqueología LXVII, pp. 45-68.

BERGER-TAL, O. \& S. BAR-DAVID (2015): «Recursive movement patterns: Review and synthesis across species», Ecosphere 6/9, art. 149. http://dx.doi.org/10.1890/ES15-00106.1.

Bermúdez, J. L. (2003): Thinking Without Words. New York: Oxford University Press. https://doi.org/10.1093/acprof:oso/9780195159691.001.0001.

BERWICK, R. C., K. OKANOYA, K., G. BECKERS \& J. J. BOlHUIS (2011): «Songs to syntax: the linguistics of birdsong», Trends in Cognitive Sciences 15/3, pp. 113-121. https://doi.org/10.1016/j.tics.2011.01.002.

BERWICK, R. C., G. BECKERS, K. OKANOYA \& J. J. Bolhuis (2012): «A bird's eye view of human language evolution», Frontiers in Evolutionary Neuroscience 4, art. 5, pp. 1-25. https://doi.org/10.3389/fnevo.2012.00005.

BiCKerton, D. (1990): Language and Species. Chicago: University of Chicago Press. Cit. por Lenguaje y especies. Madrid: Alianza, 1994. 
BICKERTON, D. (1998): «Catastrophic evolution: The case for a single step from protolanguage to full human language», en J. Hurford, M. Studdert-Kennedy \& C. Knight (eds.): Approaches to the Evolution of Language. Social and Cognitive Bases. Cambridge: Cambridge University Press, pp. 341-358.

Bloom, P. (2000): How Children Learn the Meanings of Words. Cambridge (MA): MIT Press. https://doi.org/10.7551/mitpress/3577.001.0001.

Blumberg, M. (2005): Basic Instinct. The Genesis of Behavior. New York: Thunder's Mouth Press.

Bolhuis, J. J. \& M. Everaert (eds.) (2013): Birdsong, Speech, and Language. Exploring the Evolution of Mind and Brain. Cambridge (MA): MIT Press. https://doi.org/10.7551/mitpress/9322.001.0001.

BolHuis, J. J., K. OKANOYA \& C. SCHARFF (2010): «Twitter evolution: Converging mechanisms in birdsong and human speech», Nature Reviews Neuroscience 11, pp. 747-759. https://doi.org/10.1038/nrn2931.

Bolhuis, J. J., I. TATTERSAll, N. Chomsky \& R. C. BerWick (2014): «How could language have evolved?», PLOS Biology 12/8, e1001934, pp. 1-6. https://doi.org/10.1371/journal.pbio.1001934.

Budiansky, S. (1998): If a Lion Could Talk. Animal Intelligence and the Evolution of Consciousness. New York: Free Press. Cit. por Si los animales hablaran...no les entenderíamos. La evolución de la conciencia y la inteligencia. Madrid: Ateles, 2001.

BuEno-Guerra, N. \& F. Amici (eds.) (2018): Field and Laboratory Methods in Animal Cognition. A Comparative Guide. Cambridge: Cambridge University Press. https://doi.org/10.1017/9781108333191.

CARruthers, P. (2006): The Architecture of the Mind. Massive Modularity and the Flexibility of Thought. New York: Oxford University Press.

ChitTKA, L. (2017): «Bee cognition», Current Biology 27/19, pp. R1049-R1053. https://doi.org/10.1016/j.cub.2017.08.008.

Снов, J. C. (ed.) (2019): Encyclopedia of Animal Behavior, 2ª ed. Vol. 1: Overview Essays, Historical Overviews, Animal Welfare and Conservation, Cognition, Communication. Amsterdam: Elsevier.

Cholewiak, D. M., R. S. SousA-Lima \& S. CERChio (2013): «Humpback whale song hierarchical structure: Historical context and discussion of current classification issues», Marine Mammal Science 29/3, pp. E312-E332. https://doi.org/10.1111/mms.12005.

Cномsку, N. (1957): Syntactic Structures. The Hague: Mouton. https://doi.org/10.1515/9783112316009.

Сномsку, N. (1959): «A review of B. F. Skinner's Verbal Behavior», Language 35, pp. 26-58. https://doi.org/10.2307/411334.

Сномsку, N. (2017): «The language capacity: Architecture and evolution», Psychonomic Bulletin \& Review 24, pp. 200-203. https://doi.org/10.3758/s13423-016-1078-6.

CouzIN, I. D. (2009): «Collective cognition in animal groups», Trends in Cognitive Sciences 13/1, pp. 36-43. https://doi.org/10.1016/j.tics.2008.10.002.

DAWKINS, R. (1976): The Selfish Gene. Oxford: Oxford University Press.

DAWKINS, R. \& J. R. KREBS (1978): «Animal signals: Information or manipulation?», en J. R. Krebs \& N. B. Davies (eds.): Behavioural Ecology: An Evolutionary Approach. Sunderland (MA): Sinauer, pp. 282-309.

DEACon, T. (1997): The Symbolic Species. The Co-Evolution of Language and the Human Brain. London: Penguin.

Edelman, G. M. (2004): Wider Than the Sky. The Phenomenal Gift of Consciousness. New Haven: Yale University Press. https://doi.org/10.1172/ICI23795. 
Edelman, G. M., J. A. GAlly \& B. J. BAARS (2011): «Biology of consciousness», Frontiers in Psychology 2: art. 4. https://doi.org/10.3389/fpsyg.2011.00004.

English, M., M. Ancrenaz, G. Gillespie, B. Goossens, S. Nathan \& W. Linklater (2014): «Foraging site recursion by forest elephants Elephas maximus borneensis». Current Zoology 60/4, pp. 551-559. https://doi.org/10.1093/czoolo/60.4.551.

FISHER, S. E. \& S. C. VERNES (2015): «Genetics and the language sciences», Annual Review of Linguistics 1, pp. 289-310. https://doi.org/10.1146/annurev-linguist-030514-125024.

GALLISTEL, R. (1998): «Symbolic processes in the brain: The case of insect navigation», en D. Scarborough \& S. Sternberg (eds.): An Invitation to Cognitive Science. Vol. 4: Conceptual and Methodological Foundations, $2^{\text {nd }}$ ed. Cambridge (MA): MIT Press, pp. 1-51.

GotTlieB, G. (1971): Development of Species Identification in Birds: An Inquiry Into the Prenatal Determinants of Perception. Chicago: University of Chicago Press.

GotTlieb, G. (1992): Individual Development and Evolution. The Genesis of Novel Behavior. New York: Oxford University Press.

GotTlieb, G. (1997): Synthesizing Nature-Nurture: Prenatal Roots of Instinctive Behavior. Mahwah, NJ: Lawrence Erlbaum.

GYÖRI, G. (1995): «Animal communication and human language: Searching for their evolutionary relationship», en S. Puppel (ed.): The Biology of Language. Amsterdam: John Benjamins, pp. 99-126. https://doi.org/10.1075/Z.75.08gyo.

HAUSER, M. D. (1996): The Evolution of Communication. Cambridge (MA): MIT Press.

HAUSER, M. D. (2000): Wild Minds. What Animals Really Think. New York: Henry Holt. Cit. por Mentes salvajes. ¿Qué piensan los animales? Buenos Aires: Granica.

HAUSER, M. D., N. Chomsky \& W. T. Fitch (2002): «The faculty of language: What is it, who has it, and how did it evolve?», Science 298, pp. 1569-1579. https://doi.org/10.1126/science.298.5598.1569.

HAuSER, M. D. \& M. Konishi (eds.) (1999): The Design of Animal Communication. Cambridge (MA): The MIT Press.

HAUSER, M. D. \& J. WATUMULL (2017): «The universal generative faculty: The source of our expressive power in language, mathematics, morality, and music», Journal of Neurolinguistics 43/part B, pp. 78-94. https://doi.org/10.1016/i.jneuroling.2016.10.005.

Hоскетт, C. F. (1942): «A system of descriptive phonology», Language 18/1, pp. 3-21. https://doi.org/10.2307/409073.

HockeTt, C. F. (1958): A Course in Modern Linguistics. New York: MacMillan. Cit. por Curso de linguistica moderna. Buenos Aires: Eudeba, 1971.

HoRnsteIn, N. (2009): A Theory of Syntax. Minimal Operations and Universal Grammar. New York: Cambridge University Press. https://doi.org/10.1017/CB09780511575129.

HURFoRD, J. R. (2012): The Origins of Grammar. Language in the Light of Evolution, vol. II. New York: Oxford University Press.

Jablonka, E. \& M. LAmB (2005): Evolution in Four Dimensions. Genetic, Epigenetic, Behavioral, and Symbolic Variation in the History of Life. Cambridge (MA): MIT Press.

JANIK, V. \& P. J. B. SLATER (1997): «Vocal learning in mammals», Advances in the Study of Behavior 26, pp. 5999. https://doi.org/10.1016/S0065-3454(08)60377-0. 
Johnston, T. \& L. EdWARDS (2002): «Genes, interactions, and the development of behavior», Psychological Review 109/1, pp. 26-34. https://doi.org/10.1037/0033-295X.109.1.26.

KAMINSKI, J., J. CALL \& J. FISHER (2004) «Word learning in a domestic dog: Evidence for 'fast mapping'», Science 304, pp. 1682-1683. https://doi.org/10.1126/science.1097859.

KING, B. J. (2013): «When animals mourn», Scientific American, July, pp. 63-67. https://doi.org/10.1038/scientificamerican0713-62.

LAI, C., S. Fisher, J. HuRST, F. VARgha-Khadem \& A. MonaCo (2001): «A forkhead domain gene is mutated in a severe speech and language disorder», Nature 413, pp. 519-523. https://doi.org/10.1038/35097076.

LATTENKAMP, E. Z. \& S. C. VERNES (2018): «Vocal learning: A language-relevant trait in need of a broad crossspecies approach», Current Opinion in Behavioral Sciences 21, pp. 209-215. https://doi.org/10.1016/j.cobeha.2018.04.007.

LONGA, V. M. (2006): «Sobre el significado del descubrimiento del gen FOXP2», Estudios de Lingüística. Universidad de Alicante 20, pp. 177-207. https://doi.org/10.14198/ELUA2006.20.09.

LONGA, V. M. (2012): Lenguaje humano y comunicación animal. Análisis comparativo. Bucaramanga: Universidad Industrial de Santander.

LONGA, V. M. (2013): «Bibliografía seleccionada y comentada sobre comunicación y semiosis animal», Moenia. Revista Lucense de Lingüística \& Literatura 19, pp. 523-573.

LonGA, V. M. (2014): «El segundo error de Descartes: representaciones mentales en insectos», Representaciones. Revista de Estudios sobre Representación en Arte, Ciencia y Filosofía 10/1, pp. 63-87.

LONGA, V. M. (2015): «Los sistemas combinatorios animales no tienen dualidad: Hockett tenía razón», Pragmalingüística 23, pp. 122-141. https://doi.org/10.25267/Pragmalinguistica.2015.i23.07.

LONGA, V. M. \& J. J. LÓPEZ RIVERA (2005): «¿Pueden adquirir palabras los animales? Sobre el aprendizaje de palabras por un perro», Estudios de Lingüística. Universidad de Alicante 19, pp. 301-217. https://doi.org/10.14198/ELUA2005.19.15.

LONGA, V. M. \& G. LORENZO (2012): «Theoretical linguistics meets development: Explaining FL from an epigeneticist point of view», en C. Boeckx, M丷. C. Horzo Chéliz \& J. L. Mendívil Giró (eds.), Language, From a Biological Point of View. Current Issues in Biolinguistics. Newcastle Upon Tyne: Cambridge Scholars Publishing, pp. 52-84.

LONGA, V. M. \& G. LORENZO (2019): «The study of instinct», en T. K. Shackelford \& V. A. Wicker-Shackelford (eds.): Encyclopedia of Evolutionary Psychological Science. Berlin: Springer, pp. 1-4. https://doi.org/10.1007/978-3-319-16999-6 1346-1.

LoREnzo, G. \& V. M. LonGA (2018): El innatismo. Orígenes, variaciones y vitalidad de una idea. Madrid: Cátedra.

MARLER, P. (1998): «Animal communication and human language», en N. G. Jablonski \& L. C. Aiello (eds.): The Origin and Diversification of Language. San Francisco: California Academy of Sciences, pp. 1-19.

Marler, P. \& H. Slabbekoorn (eds.) (2004): Nature's Music. The Science of Birdsong. San Diego: Elsevier Academic Press.

MATHER, J. A. (2008): «Cephalopod consciousness: Behavioural evidence». Consciousness and Cognition 17/1, pp. 37-48. https://doi.org/10.1016/i.concog.2006.11.006.

MEIJER, E. (2019): Animal Languages. The Secret Conversations of the Living World. London: John Murray. Vers. original en holandés: Dierentalen. Leusden: ISVW, 2016. https://doi.org/10.18574/nyu/9781479859351.003.0003. 
Menzel, R. \& J. Fisher (eds.) (2011): Animal Thinking. Contemporary Issues in Comparative Cognition. Cambridge (MA): MIT Press. https://doi.org/10.7551/mitpress/9780262016636.001.0001.

Moore, D. S. (2001): The Dependent Gene. The Fallacy of 'Nature vs. Nurture'. New York: Henry Holt.

Moss, L. (2003): What Genes Can't Do. Cambridge (MA): MIT Press. https://doi.org/10.7551/mitpress/7205.001.0001.

Moynihan, M. F. (1985): Communication and Non-Communication by Cephalopods. Bloomington: Indiana University Press.

Oller, D. K. \& U. GRIEBEL (eds.) (2004): Evolution of Communication Systems: A Comparative Approach. Cambridge (MA): MIT Press. https://doi.org/10.7551/mitpress/2879.001.0001.

OUATTARA, K., A. LEMASSON \& K. ZuBERbÜHLER (2009): «Campbell monkeys use affixation to alter call meaning», PLoS One 4/11: e7808. https://doi.org/10.1371/journal.pone.0007808.

Owings, D. H. \& E. S. MorTon (1998): Animal Vocal Communication: A New Approach. Cambridge: Cambridge University Press. https://doi.org/10.1017/CB09781139167901.

OWREN, M. J. D. RENDALL \& M. J. RYAN (2010): «Redefining animal signaling: influence versus information in communication», Biology \& Philosophy 25, pp. 755-780. https://doi.org/10.1007/s10539-010-9224-4.

PAYNe, R. \& S. MCVAY (1971): «Songs of humpback whales», Science 173, pp. 585-597. https://doi.org/10.1126/science.173.3997.585.

Pepperberg, I. M. (1999): The Alex Studies. Cognitive and Communicative Abilities of Grey Parrots. Cambridge (MA): Harvard University Press.

PILlEY, J. \& A. REID (2011): «Border collie comprehends object names as verbal referents», Behavioural Processes 86/2, pp. 184-195. https://doi.org/10.1016/j.beproc.2010.11.007.

QUINE, W. V. O. (1960): Word and Object. Cambridge (MA): MIT Press. Cit. por Palabra y objeto. Barcelona: Herder, 2001.

RENDALL, D., M. J. OWREN \& M. J. RYAn (2009): «What do animal signals mean?», Animal Behaviour 78, pp. 233240. https://doi.org/10.1016/j.anbehav.2009.06.007.

ReZnikova, Z. (2018): «Ants: Individual and social cognition», en N. Bueno-Guerra \& F. Amici (eds.): Field and Laboratory Methods in Animal Cognition. A Comparative Guide. Cambridge: Cambridge University Press, pp. 8-30. https://doi.org/10.1017/9781108333191.003.

RondAL, J. A. (2000): Le langage: de l'animal aux origines du language humain. Sprimont: Mardaga.

SAUSSURE, F. de (1916) [ed. por C. Bally \& A. Sechehaye]: Cours de linguistique générale. Paris: Payot.

Savage-Rumbaugh, S. \& S. G. Shanker \& T. J. Taylor (1998): Apes, Language, and the Human Mind. Oxford: Oxford University Press.

Segerdahl, P. \& W. Fields \& S. SaVage-Rumbaugh (2005): Kanzi's Primal Language. The Cultural Initiation of Primates Into Language. Houndmills: Palgrave Macmillan. https://doi.org/10.1057/9780230513389.

Seyfarth, R. M., D. L. Cheney, T. Bergman, J. Fisher, K. ZuberbÜHler \& K. HAmmerschmidt (2010): «The central importance of information in studies of animal communication», Animal Behaviour 80, pp. 3-8. https://doi.org/10.1016/i.anbehav.2010.04.012.

SEYFARTH, R., D. CHENEY \& P. MARLER (1980a): «Monkey responses to three different alarm calls: evidence of predator classification and semantic communication». Science 210, pp. 801-803. https://doi.org/10.1126/science.7433999. 
SEYFARTH, R., D. ChENEY \& P. MARLER (1980b): «Vervet monkeys alarm calls: semantic communication in a free-ranging primate». Animal Behaviour 28, pp. 1070-1094. https://doi.org/10.1016/S00033472(80)80097-2.

Simone, R. (1990): Fondamenti di linguistica. Roma: Gius, Laterza \& Figli. Cit. por Fundamentos de lingüística. Barcelona: Ariel, 1993.

Slater, P. J. B. (1999): Essentials of Animal Behaviour. Cambridge: Cambridge University Press. Cit. por El comportamiento animal. Madrid: Cambridge University Press, 2000.

SlobodchiкоfF, C. (2012): Chasing Doctor Dolittle. Learning the Language of Animals. New York: St. Martin's Press.

SLOBODCHIKOFF, C., J. KIRIAZIS, C. FISHER \& E. CREEF (1991): «Semantic information distinguishing individual predators in the alarm calls of Gunnison's prairie dogs», Animal Behaviour 42, pp. 712-719. https://doi.org/10.1016/S0003-3472(05)80117-4.

Slobodchikoff, C., A. PASEKA \& J. L. Verdolin (2009): «Prairie dog alarm calls encode labels about predator colors», Animal Cognition 12/3, pp. 435-439. https://doi.org/10.1007/s10071-008-0203-y.

SMITH, C. L. \& J. JoHnSON (2012): «The chicken challenge: What contemporary studies of fowl mean for science and ethics», Between The Species 15/1, pp. 75-102. https://doi.org/10.15368/bts.2012v15n1.4.

SPELKE, E. \& S. A. LEE (2012): «Core systems of geometry in animal minds», Philosophical Transactions of the Royal Society B 367, pp. 2784-2793. https://doi.org/10.1098/rstb.2012.0210.

Stoeger, A. S., D. Mietchen, S. OH, S. De Silva, C.T. HerbST, S. Kwon \& W. T. Fitch (2012): «An Asian elephant imitates human speech», Current Biology 22/22, pp. 2144-2148. https://doi.org/10.1016/i.cub.2012.09.022.

SUZUKI, R., J. R. BUCK \& P. L. TYACK (2006): «Information entropy of humpback whale songs», Journal of the Acoustic Society of America 119/3, pp. 1849-1866. https://doi.org/10.1121/1.2161827.

Suzuki, T. N. \& K. ZuberbÜHLer (2019): «Animal syntax», Current Biology 29/14, pp. R669-R671. https://doi.org/10.1016/j.cub.2019.05.045.

TEMPLETON, C. N., E. GREENE \& K. DAVIS (2005): «Allometry of alarm calls: Black-capped chickadees encode information about predator size», Science 308, pp. 1934-1937. https://doi.org/10.1126/science.1108841.

Tomasello, M. (2003): Constructing a Language. A Usage-Based Theory of Language Acquisition. Cambridge (MA): Harvard University Press.

VAN GulicK, R. (2018): «Consciousness», en E. N. Zalta (ed.): The Stanford Encyclopedia of Philosophy. Accesible en: https://plato.stanford.edu/entries/consciousness/\#ConCon [acceso: 04/05/2020].

WAtumulL, J., M. D. HAuSER, I. G. RoBerTs \& N. HoRnstein (2014): «On recursion», Frontiers in Psychology 4: art. 1017, pp. 1-7. https://doi.org/10.3389/fpsyg.2013.01017.

WebB, B. (2012): «Cognition in insects», Philosophical Transactions of the Royal Society B 367, pp. 27152722. https://doi.org/10.1098/rstb.2012.0218.

West-Eberhard, M. (2003): Developmental Plasticity and Evolution. New York: Oxford University Press.

Wynne, C. D. L. \& M. A. R. Udell (2013): Animal Cognition. Evolution, Behavior and Cognition, 2nd ed. London: Palgrave Macmillan.

ZUBERBÜHLER, K. (2002): «A syntactic rule in forest monkey communication», Animal Behaviour 63, pp. 293299. https://doi.org/10.1006/anbe.2001.1914. 


\section{NOTAS}

1 Trabajo realizado en el marco del proyecto “Desarrollo, adquisición y mecanismos de variación lingüística” (FFI201787699-P), financiado por el Ministerio de Economía, Industria y Competitividad. Deseo reconocer y agradecer los comentarios y sugerencias muy útiles de dos revisores anónimos de Verba.

${ }^{2}$ Ya en la contraportada se afirma que la obra «will show you how to talk (a little better) with the animals».

3 La propia Meijer señala que "the words that Chaser learned are tied to objects" (pp. 97-88), de modo que "comprehension means comprehension within the context of fetching and categorization of objects, not the learning of abstract concepts" (p. 98).

4 Slobodchikoff et alii (1991) habían empleado humanos con camisetas de cuatro colores, pero el sexo y tamaño de los humanos diferían, por lo que Slobodchikoff et alii (2009) unifican esas variables para determinar únicamente las diferencias entre las llamadas asociadas al color de las camisetas.

5 Un revisor de Verba refiere que, muy recientemente, el propio Zuberbühler ha rebajado su alegato a favor de la existencia de sintaxis en esa especie, indicando que «better evidence for syntax-like structure is provided by studies of bird calls» (Suzuki \& Zuberbühler 2019: R669). 